\title{
Improved Method for Converting Equivalent Sand-grain Roughness to Hazen-Williams Coefficient
}

\author{
Thomas M. Adams \\ Rose-Hulman Institute of Technology \\ 5500 Wabash Ave., Terre Haute, IN, USA \\ adams1@ rose-hulman.edu
}

\begin{abstract}
A method of predicting the Hazen-Williams roughness coefficient from knowledge of equivalent sand-grain roughness is presented. An equation resulting from combining the Hazen-Williams, Darcy-Weisbach, and Colebrook-White equations is used as the basis of the relationship. This equation in turn is used as the theoretical foundation for correlating existing experimental data for sandgrain roughness and Hazen-Williams coefficient. Previously developed methods have not incorporated such data. The result is a correlation that shows very good agreement between accepted and predicted values, providing an improved means by which conversion of sand-grain roughness to Hazen-Williams coefficient can be accomplished where values for one or the other are not readily available.
\end{abstract}

Keywords: Hazen-Williams coefficient, equivalent sand-grain roughness, pipe flow

\section{Introduction} given by

In finding the head loss in turbulent pipe flow, the customary approach is to make use of the Darcy-Weisbach equation

$$
h_{L}=f \frac{L}{D} \frac{\rho V^{2}}{2 g}
$$

where $h_{L}$ is the total head loss, $L$ is pipe length, $D$ is diameter, $\rho$ is density, $V$ is velocity, $g$ is gravitational acceleration, and $f$ is the Darcy friction factor. The Darcy friction factor in turn is a function of Reynolds number and relative roughness. That is

$$
f=f\left(\operatorname{Re}, \frac{\varepsilon}{D}\right)
$$

where $R e$ is Reynolds number and $\varepsilon$ is the equivalent sand-grain roughness of the pipe surface. The friction factor is typically found by making use of the Colebrook-White equation or its graphical representation given by the Moody chart.

A number of empirical relationships are also common in the analysis and modelling of pipe flow, the Hazen-Williams equation representing perhaps the most widely used [1]. The equation is given by [2]

$$
V=K_{H W} C R_{h}^{0.63} S^{0.54}
$$

where $V$ is fluid velocity, $C$ is the Hazen-Williams coefficient, $R_{h}$ is the hydraulic radius, defined as the cross-sectional area divided by the wetted perimeter $\left(R_{h}=A / P\right)$, and $S$ is the slope of the energy grade line, or the total head loss per unit length of pipe $\left(S=h_{L} / L\right)$. The constant $K_{H W}$ is a dimensional constant with a value of $0.849 \mathrm{~m}^{0.37} / \mathrm{s}$ in the SI system. The Hazen-Williams coefficient $C$ characterizes the roughness of the pipe, with values ranging from 140 and above for very smooth pipes, to 60 for badly corroded pipes [1]. Used extensively in the United States in the design of water-supply systems [3], the equation was originally developed for water flow in larger pipes (diameters greater than $5 \mathrm{~cm}$ ) and lower velocities 
(lower than $3 \mathrm{~m} / \mathrm{s}$ ) [4]. In practice, however, it appears that engineers do use the Hazen-Williams equation for smaller diameters [5] and larger velocities [6], though it is restricted for use with water and not with other fluids [1, 4].

A major advantage of the Hazen-Williams equation over the Darcy-Weisbach equation is in solving Type II and Type III pipe flow problems in which flow rate and pipe diameter are unknowns, respectively. Use of Eq. (1) allows for velocity or diameter (via hydraulic radius) to be solved for directly, whereas use of the Darcy friction factor requires an iterative procedure. Furthermore, references such as [5] and [6] give extensive values for $C$ that also take into account the effective roughening of a pipe surface due to corrosion over time. Disadvantages include its use being limited only to water, and the lack of a theory behind its form.

\section{Conversion Method}

For cases in which information about only one roughness parameter is available and one wishes to employ the opposite pipe flow equation, a reliable method to convert roughness parameters is desirable. Walski et al. [7] outline such a method based on eliminating head loss per unit length between the flow equations and assuming a water velocity of $0.9 \mathrm{~m} / \mathrm{s}$ as a representative value. This approach gives a somewhat "theoretical" relationship between $\varepsilon$ and $C$. In the present work velocity is eliminated between the flow equations instead, and the resulting equation is used as the basis of correlating existing accepted values of $\varepsilon$ and $C$ to one another.

\subsection{Derivation of Conversion Equation}

The Colebrook-White equation relating Darcy friction factor to Reynolds number and relative roughness is given by

$$
\frac{1}{\sqrt{f}}=1.74-0.869 \ln \left\lfloor\frac{2 \varepsilon}{D}+\frac{18.7}{\operatorname{Re} \sqrt{f}}\right\rfloor
$$

Using the definition of Reynolds number and solving Eq. 1 for $f$ and then substituting into Eq. 4 yields after much manipulation

$$
V=-1.229 \sqrt{D g S} \ln \left[\frac{\varepsilon}{3.7 D}+\frac{1.785 v}{D} \sqrt{\frac{1}{D g S}}\right],
$$

where $v$ is the kinematic viscosity. Equating Eq. 5 with the Hazen-Williams relation given by Eq. 3, and recognizing that $D=4 R_{h}$, results in the following expression for $C$ :

$$
C=-K_{1} \sqrt{g} D^{-0.13} S^{-0.04} \ln \left[\frac{\varepsilon}{3.7 D}+\frac{1.785 v}{D} \sqrt{\frac{1}{D g S}}\right],
$$

where $K_{1}=3.46 \mathrm{~s} / \mathrm{m}^{0.37}$.

Further simplifications can be made by assuming wholly turbulent flow, which makes the second term within the natural logarithm small compared to the first, and additionally assuming that the head loss per unit length is dominated by diameter and is therefore directly proportional to it. This gives

$$
C=-K_{1} \sqrt{g} D^{-0.13} K_{2} D^{n} \ln \left\lfloor\frac{\varepsilon}{3.7 D}\right\rfloor,
$$

where $K_{2}$ is an additional dimensional constant and the exponent $n$ is employed in place of 0.04 in order to give more flexibility in curve fitting roughness data. We would expect, however, that $n$ should be on the order of 0.04 . 


\subsection{Determination of Equation Constants}

Equation (7) was used to relate the extensive $C$ and $\varepsilon$ data compiled in [5] in order to optimize the $K_{2}$ and $n$ parameters. The specific materials and roughness values were chosen so as to minimize the ambiguity between $\varepsilon$ and the corresponding values of $C$, and to span a large range of sand-grain roughness over several orders of magnitude. The specific values used in this study are given in Table 1.

Table 1: Values of sand-grain roughness and Hazen-Williams coefficient used in parameter optimization. (Taken from Lamont [5].)

\begin{tabular}{|l|l|c|c|c|c|c|c|}
\hline \multirow{2}{*}{\multicolumn{1}{|c|}{ Material }} & $\varepsilon$ & \multicolumn{6}{c|}{$C$ values at various pipe diameters } \\
\cline { 5 - 9 } & {$[\mathrm{mm}]$} & $2.5 \mathrm{~cm}$ & $7.6 \mathrm{~cm}$ & $15.2 \mathrm{~cm}$ & $30.5 \mathrm{~cm}$ & $61 \mathrm{~cm}$ & $122 \mathrm{~cm}$ \\
\hline Wrought iron & 0.05 & 129 & 137 & 142 & & & \\
\hline Coated cast iron & 0.125 & & 129 & 133 & 138 & 140 & 141 \\
\hline Uncoated cast iron & 0.25 & & 121 & 125 & 130 & 132 & 134 \\
\hline Concrete (Scobey Class 3) & 0.50 & & 109 & 116 & 121 & 125 & 127 \\
\hline Concrete (Scobey Class 2) & 1.25 & & 95 & 102 & 106 & 110 & 113 \\
\hline
\end{tabular}

A least squares fit of the data in Table 1 as correlated by Eq. 7 yields values of $K_{2}=1.30 \mathrm{~m}^{-0.062}$ and $n=0.062$. Substitution of these values into Eq. (7) gives

$$
C=-A \sqrt{g} D_{h}^{-0.068} \ln \left\lfloor\frac{\varepsilon}{3.7 D}\right\rfloor,
$$

where $A=K_{1} K_{2}=4.438 \mathrm{~s} / \mathrm{m}^{0.432}$.

\section{Results and Discussion}

Figure 1 shows a comparison between the accepted values of Hazen-Williams coefficient as given in Lamont [5] and values as predicted by Eq. 8 for various sand-grain roughnesses and pipe diameters. For the range of roughness and diameters shown here, the predicted values are in very good agreement with the accepted values. The larger discrepancies seen at lower values of sand-grain roughness would likely not contribute to significant calculation errors when using the Hazen-Williams equation, since the values of $C$ correspond to very smooth pipes.

A correlation developed by Walski et al. [7] to predict $C$ based on sand-grain roughness is given by

$$
C=18.0-37.2 \log \left\lfloor\frac{\varepsilon}{D}\right\rfloor
$$

Both Eqs. 8 and 9 result from inferring a relationship between $C$ and $\varepsilon$ based on Eqs. 1 and 3, and both may be deemed semiempirical. In the present work, however, the resulting equation is used to correlate $C$ and $\varepsilon$ data, whereas Eq. 9 seeks to predict $C$ based only on the resulting relationship without incorporating such information. In other words Eq. 9 predicts what $C$ ought to be for a given $\varepsilon$, whereas Eq. 8 is an attempt to well-correlate actually known values of $C$ and $\varepsilon$ to one another. It should also be noted that Walski et al. [7] assume a representative water velocity of $V=0.9 \mathrm{~m} / \mathrm{s}$ in order to derive the final form of Eq. 9. The derivation of Eq. 8 does not make any explicit assumption regarding velocity, though it may certainly be true that it serves as a better prediction tool at particular velocities then at others. 

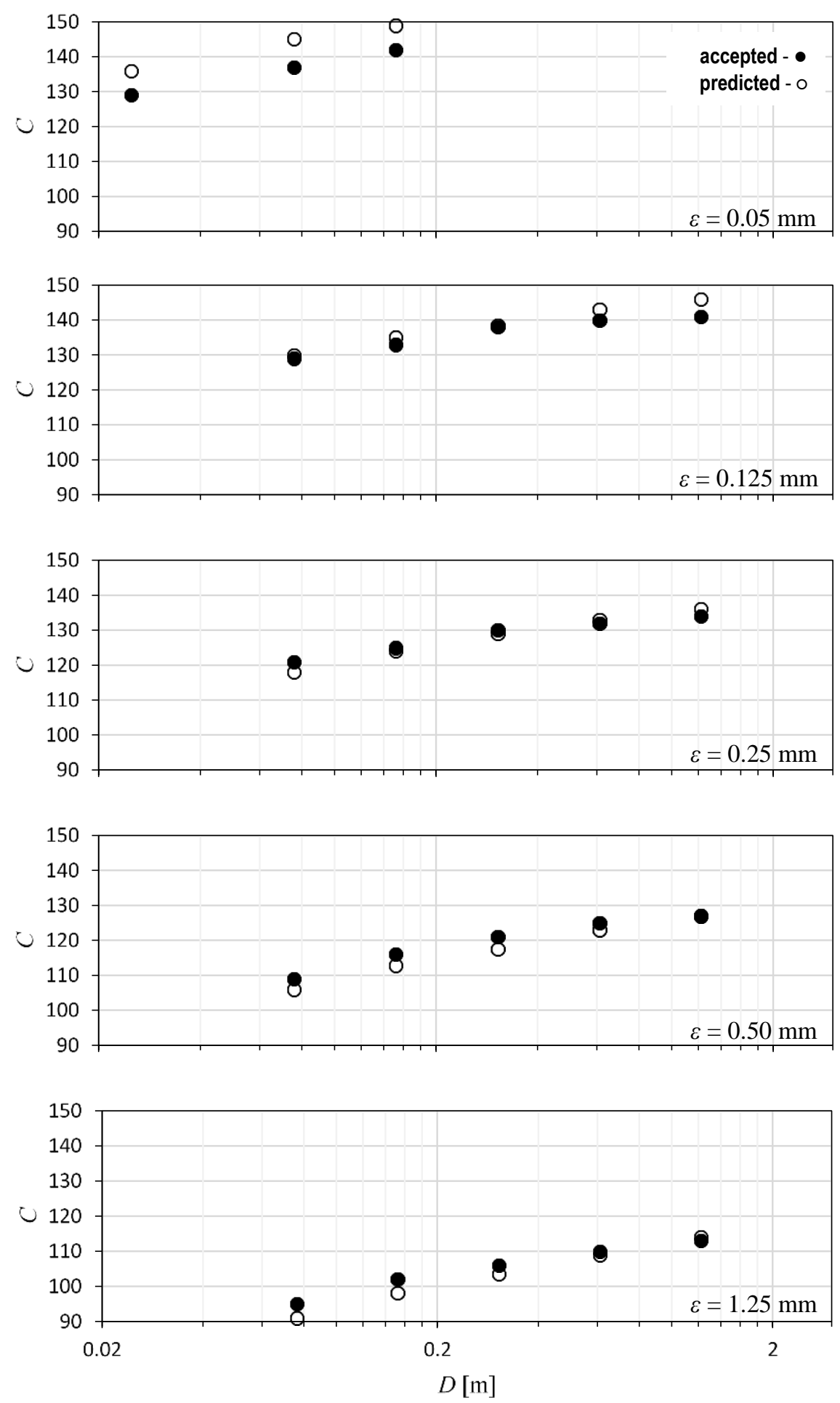

Fig. 1: Comparison of converted values of Hazen-Williams coefficient to accepted values for various sand-grain roughnessess. 
Figure 2 shows predictions for $C$ as given by Eqs. 8 and 9 plotted against the accepted values for the entire range of $\varepsilon$ and $D$ values given in Table 1. A perfect prediction would fall on the solid line, which has a slope of one. The two dotted lines enclose the accepted value of $C$ to $\pm 5 \%$.

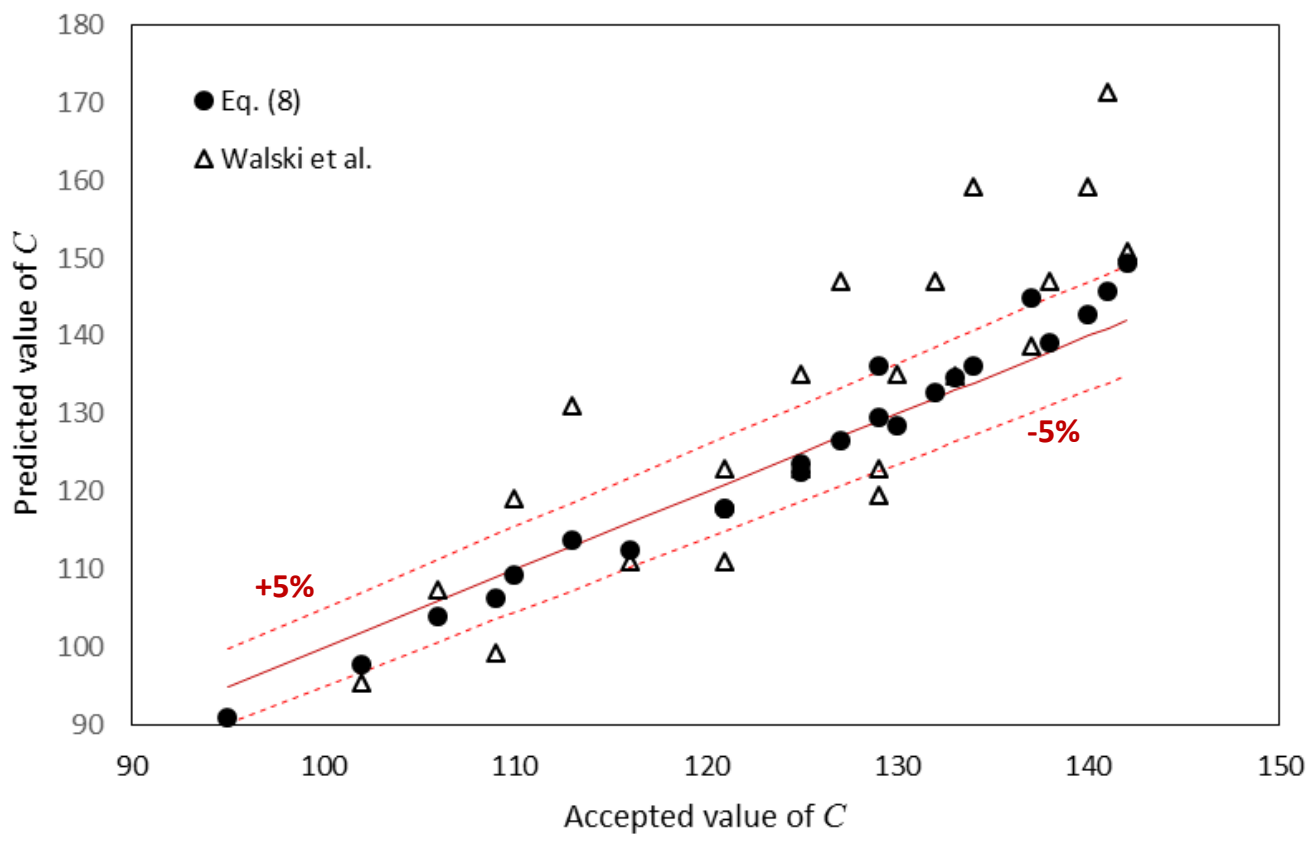

Fig. 2: Comparison of prediction methods for $C$ as given by Eq. 8 and Walski et al. [7].

Figure 2 shows that Eq. 8 well predicts $C$ based on $\varepsilon$ and $D$, giving an improved estimate over that of Eq. 9. The average absolute value of error for the predictions based on Eq. 8 is $2.3 \%$, with the largest error falling just under $6 \%$. The corresponding values for average and largest error when using Eq. (9) are 7\% and 18\%, respectively. Naturally the use of Eq. 8 should be limited to the ranges of $\varepsilon$ and $D$ which were employed in its development.

\section{Conclusion}

An improved method of predicting the Hazen-Williams coefficient from knowledge of equivalent sand-grain roughness has been presented. The method is based on a relation resulting from combining the Hazen-Williams, DarcyWeisbach, and the Colebrook-White equations. This relation serves as the basis of a parameter optimization scheme for correlating existing experimental data for sand-grain roughness and Hazen-Williams coefficient. For the range of sand-grain roughnesses investigated, $\varepsilon=0.05-1.25 \mathrm{~mm}$, predicted values of Hazen-Williams coefficient show an average absolute value of deviation of $2.3 \%$ from the accepted published values, with the highest deviation not exceeding $6 \%$. The developed relation provides more accurate estimates for Hazen-Williams roughness values than do previous methods, which do not incorporate experimental data for roughness. The correlation should provide a useful tool for the conversion of roughness values where data are not readily available.

\section{Acknowledgements}

The author wishes to thank Tomás Jimenez for inspiring the idea on which this research is based.

\section{References}

[1] R. M. Olson and S. J. Wright, Essential of Engineering Fluid Mechanics, ${ }^{\text {th }}$ Ed. St. Louis, MO: Harper and Row, Publishers, 1990.

[2] G. S. Williams and A. Hazen, Hydraulics Tables. New York: John Wiley and Sons, 1920. 
[3] L. A. Mays, Water Resources Engineering, $2^{\text {nd }}$ Ed. New York: John Wiley and Sons, 2011.

[4] R. J. Houghtalen, N. H. C. Hwang, and A. O. H. Akan, Fundamentals of Hydraulic Engineering Systems, ${ }^{\text {th }}$ Ed. Saddle River NJ: Prentice Hall, 2010.

[5] P. A. Lamont, "Common pipe flow formulas compared with the theory of roughness" J. Am. Water Works Assoc., vol. 73 , no. 5, pp. 274-280, 1981.

[6] T. M. Walski, D. V. Chase, and W. Grayman, "Modeling Theory," in Advanced Water Distribution Modeling and Management. Exton, PA: Bentley Institute Press, 2007, pp. 19-74.

[7] T. M. Walski, W. W. Sharp, and F. D. Shields, "Predicting Internal Roughness in Water Mains," Miscellaneous Paper EL-88-2, US Army Engineer Waterways Experiment Station, Vicksburg, MS, 1988. 